\title{
Factors Affecting the Usability of Nasal Drug Delivery Devices in Children
}

\author{
Mikio Murata ${ }^{1 *}$, Mayumi Muta ${ }^{1}$, Chiori Murakami ${ }^{1}$, Akio Nanba ${ }^{1}$, Kiyoshi Kubota ${ }^{1}$, Masaho \\ Hayashi $^{1}$, Kiyomi Sadamoto ${ }^{2}$ and Masahiro Sumi ${ }^{1}$ \\ ${ }^{1}$ Department of Clinical Pharmacy, Yokohama University of Pharmacy, Japan
}

${ }^{2}$ Research Center, Shonan University of Medical Sciences, Japan

*Corresponding author: Mikio Murata, Department of Clinical Pharmacy, Yokohama University of Pharmacy, Japan.

To Cite This Article: Mikio Murata, Mayumi Muta, Chiori Murakami, Akio Nanba, Factors Affecting the Usability of Nasal Drug Delivery Devices in Children. Am J Biomed Sci \& Res. 2021 - 12(1). AJBSR.MS.ID.001709. DOI: 10.34297/AJBSR.2021.12.001709.

Received: 眥 February 02, 2021; Published: 盋 February 17, 2021

\begin{abstract}
Drug product labelling, packaging, and devices directly affect medication adherence and the usability of medications. we previously performed a small-scale study and identified nasal drug delivery devices that were difficult for children to use. We previously performed a small-scale study and identified nasal drug delivery devices that were difficult for children to use. In the present study, we expanded the scale of the study to assess the usability of nasal drug delivery devices and factors that affect usability. Five metered-dose spray devices were selected (A-E); devices A-C had a volume of $30 \mathrm{~mL}$, whereas D-E had a volume of $15 \mathrm{~mL}$ per device. Among OTC medications commonly available, there are some nasal drug delivery devices that children are unable to use even though they are of the appropriate age to use the device. Physiological factors of subjects, such as age, pinch strength, grip strength, and hand/ finger size had little impact on the ease of opening the cap, ease of holding the device with three fingers, ease of pressing on the lever, or overall usability. On the other hand, the shape of the lever affected overall usability. Furthermore, the distance from bottom to lever top for devices with circular levers was correlated with the ease of holding the device with three fingers. Similarly, for devices with wing-shaped levers, the distance from bottom to lever top and the average effort force required to press on the lever affected the ease of pressing on the lever. Based on our study, marketing for OTC medications should consider the significant effects of usability on the choice of medication and that devices may not be easy to use for children. Furthermore, it is important to design a user-friendly device for OTC medications.
\end{abstract}

Keywords: Nasal Drug Delivery Devices, Adherence, Children, OTC Medications, Three Fingers, Drug Product Labelling, Generic Drugs, Pharmacists, Dynamometer, Pinch Strength, Approximately

Abbreviations: OTC medications; over-the-counter medications, CR packaging; child-resistant packaging, NRS; numeric rating scale

\section{Introduction}

In Japan, a record-breaking national healthcare expenditure of over 43 trillion yen was paid to medical institutions for insured procedures in 2018. This increase in expenditure can be attributed to the increasing elderly population, with $60.6 \%$ being used for individuals over the age of 65 [1]. By the Amount of Production by Drug Type in 2018, OTC medications comprised approximately $12 \%$ of the production market with approximately 740 billion yen in comparison to the production of prescription drugs, which was equivalent to approximately 6.2 trillion yen [2]. Expenditure on OTC medications is significantly lower in Japan than in other countries in the Organization for Economic Cooperation and Development (OECD) [3]. The national health insurance program and free access to healthcare are the important basis of care in Japan to ensure the health of the population [4]. As such, individuals often visit hospitals more often than purchase OTC medications. However, due to advancements in medical technologies and the change in common disease types due to the declining birth rate and aging society, improvements in financing of the health care system have become an important issue. To address this, various measures have been implemented to increase the prevalence of generic drugs, 
establish disease prevention and health promotion measures, and promote the use of OTC medications as a means of self-medication $[4,5]$.

Although Japan previously focused on providing complete healthcare in hospitals, social security system reform has been established to promote community-integrated medical and longterm care to use resources more effectively and appropriately [4]. Thus, OTC medications are expected to play an essential role in medical care in Japan. To promote appropriate selfmedication practice, it is important to ensure their safety, and optimize drug product labelling and the usability of a medical device and packaging [6]. In Europe and the US, many guidelines, and regulations, such as CR packaging, are used as risk control measures for drug product labelling, packaging, and devices [6,7]. Drug product labelling, packaging, and devices directly affect medication adherence and the usability of medications [8]. As OTC medications can be dispensed by pharmacists in communities, it is important to ensure that they are safe, and can be used easily among children and the elderly population [9]. OTC nasal sprays for acute rhinitis and allergic rhinitis are to be used by individuals over the age of 7 [10]; however, we previously performed a small-scale study and identified nasal drug delivery devices that were difficult

Table 1: Age, pinch strength, grip strength, and hand/ finger size of the subjects $(n=90)$.

\begin{tabular}{|c|c|c|}
\hline & Mean (SD) & Range \\
\hline Age (Month) & $115.4(18.8)$ & $85-149$ \\
\hline Pinch strength (kg) & $4.0(1.4)$ & $1-8$ \\
\hline Grip strength $(\mathrm{kg})$ & $13.9(4.3)$ & $6-23$ \\
\hline Hand length from the wrist crease (mm) & $15.0(1.2)$ & $12.3-17.5$ \\
\hline Thumb length (mm) & $5.2(0.59)$ & $3.9-6.5$ \\
\hline Palm length from the wrist crease - thumb (mm) & $6.0(0.6)$ & $4.2-7.5$ \\
\hline Palm length from the wrist crease - middle $(\mathrm{mm})$ & $8.7(0.8)$ & $6.6-11.5$ \\
\hline Hand breadth (mm) & $7.3(0.7)$ & $5.7-9.4$ \\
\hline Hand circumference (mm) & $16.1(1.3)$ & 13.4-19.6 \\
\hline
\end{tabular}

\section{Hand/ finger size, pinch strength, and grip strength}

Measurements of the dominant hand (hand length from the wrist crease, thumb length, palm length from the wrist creasethumb, palm length from the wrist crease-middle, hand breadth, and hand circumference) were made using a digital caliper (CD30C, Mitutoyo Corporation, Japan) and measuring tape (NDA-715, NIHON DOKI CO., LTD, Japan). Measurement items were selected based on the Japanese hand measurement standards developed by the National Institute of Advanced Industrial Science and Technology (AIST) [12]. Pinch strength of the dominant hand was measured using a hydraulic pinch gauge (SH5005, SAKAI Medical Co., Ltd, Japan). Grip strength of the dominant hand was measured using a digital dynamometer (Grip D TKK-5401, Takei Scientific Instruments Co., Ltd., Japan) and an average of two measurements for children to use [11]. In the present study, we expanded the scale of the study to assess the usability of nasal drug delivery devices and factors that affect usability.

\section{Materials and Methods}

A total of 90 healthy subjects aged between 7 and 12 were included after receiving consent from the subjects and their parents. The size of the hands and fingers, pinch strength, and grip strength were measured. Subjects subsequently used 5 nasal drug delivery devices (A-E) and assessed the usability of each device.

\section{Nasal drug delivery device}

OTC nasal drug delivery devices that are used to treat acute rhinitis and allergic rhinitis were included. Classification of the nasal drug delivery devices in the study. Five metered-dose spray devices were selected (A-E); devices A-C had a volume of $30 \mathrm{~mL}$, whereas D-E had a volume of $15 \mathrm{~mL}$ per device. Devices A and E were of the same drug in a different volume. To the study, the labels on the devices were covered with tape. Devices A and E were the same, but other devices had different shapes and caps. The nasal drug delivery devices used are summarized in Table 1 and the images of the devices are shown in Figure 1. were used for analysis.

\section{Measurements}

\section{Objective measurements}

Study subjects were observed for the following:

1. Subject can open the cap (Yes/ No)

2. Subject can hold the device with three fingers (Yes/ No)

3. Subject can press on the lever (Yes/ No)

\section{Sensory measurements (7-12-year-old children)}

Usability of the nasal drug delivery devices were evaluated using the NRS (0-10) for the following items:

1. Ease of opening the cap. 
2. Ease of holding the device with three fingers (thumb on the bottom of the device, index, and middle fingers on the lever)

3. Ease of pressing on the lever.

4. Overall usability

After the assessment of sensory measurements, we asked the subjects and their parents which of the devices they want to use in the future.

\section{Questionnaire for parents}

The following questions were included in the questionnaire for parents:

1. Does your child use any medications at home (Yes/ No)

2. If Yes to above, what type of medications do they use? (Please describe)

3. Does your child bring any medications to school and take it by themselves (currently or in the past)? (Yes/ No)

4. If Yes to above, do the teachers help with taking the medication? (Yes/ No)

\section{Mechanical measurements of the nasal drug delivery devices}

Effort force required to press the lever of the nasal drug delivery devices was measured over time using a digital force gauge (FGP 100: Nidec-Shimpo Corp.) and a simple force test stand (FGS100VC: Nidec-Shimpo Corp.). The test stand was set to operate at $200 \mathrm{~mm} / \mathrm{min}$ and the measurements were taken 50 times/ sec. Measurements of each device were taken 5 times.

\section{Statistical analysis}

JMP ver.11.2.1 (SAS Institute Inc.) was used for all statistical analyses.

\section{Ethical considerations}

The study was approved by the Yokohama University of Pharmacy Research Ethics Board (No. C18007). Written informed consent was received from the parents (guardians) of the subjects. In addition, informed consent was received from the study subjects after obtaining approval from their parents (guardians).

\section{Results and Discussion}

A total of 52 male and 38 female subjects were included in the study. The age, pinch strength, grip strength, and hand/ finger size, and the correlation among these assessment metrics are shown in Tables 1 and 2. The age, pinch strength, grip strength, and hand/ finger size of the subjects had weak to strong positive correlations, except between palm length from the wrist crease-thumb ( $\mathrm{mm}$ ) and pinch strength (kg) [Table 1]. During objective assessment, one subject was unable to open the cap on device C (age: 94 months, pinch strength: $2 \mathrm{~kg}$, grip strength: $8.6 \mathrm{~kg}$, hand length from the wrist crease: $12.9 \mathrm{~mm}$, thumb length: $4.7 \mathrm{~mm}$, palm length from the wrist crease-thumb: $5.2 \mathrm{~mm}$, palm length from the wrist crease-middle: $7.5 \mathrm{~mm}$, hand breadth: $6.9 \mathrm{~mm}$, and hand circumference: $14.5 \mathrm{~mm}$ ). Another subject was unable to completely press on the lever on device B (age: 88 months, pinch strength: 6kg, grip strength: $10.7 \mathrm{~kg}$, hand length from the wrist crease: $13.1 \mathrm{~mm}$, thumb length: $4.8 \mathrm{~mm}$, palm length from the wrist crease-thumb: $5.5 \mathrm{~mm}$, palm length from the wrist crease-middle: $7.5 \mathrm{~mm}$, hand breadth: $6.0 \mathrm{~mm}$, and hand circumference: $14.1 \mathrm{~mm}$ ). None of the other subjects were unable to complete the tasks. Comments collected from text observers for each item of the objective measurements are listed in Table 3.

In terms of whether subjects were able to open the cap, many subjects were either having trouble opening the cap or had to try multiple times to open the cap on device C. In terms of whether subjects were able to hold the device, subjects were barely able to hold or having trouble with holding devices A and B, which were both 30-mL devices. Device B had a wing-shaped lever, which made it easier to hold than device A, which had a circular lever. Lastly, in terms of whether subjects were able to press on the lever, many had trouble with device B. The usability of devices A-E determined by having study subjects use them is shown in Figure 2. Devices B and $\mathrm{E}$ were scored high on ease of opening the cap, whereas device $\mathrm{C}$ had the lowest score. NRS scores for device $\mathrm{C}$ were significantly lower than those of other devices. Although they had the same caps, the NRS score of device E (15mL) was significantly higher than that of device $A(30 \mathrm{~mL})$. The NRS score for the ease of holding the device with three fingers was the highest for 15-mL devices $\mathrm{D}$ and E. In contrast, the NRS score for device A was significantly lower than that of other devices. Devices A, D, and E had circular levers, whereas devices B and C had wing-shaped levers. Device A was a 30-mL device with a circular lever [Table 2].

The NRS score for the ease of pressing on the lever for the wingshaped levers was higher for device $C$ than for device B. NRS scores for device B were significantly lower than those of other devices. The overall usability NRS score was the highest for device E. The usability of 15-mL devices $\mathrm{D}$ and $\mathrm{E}$ was rated higher than that of 30-mL devices $\mathrm{A}, \mathrm{B}$, and $\mathrm{C}$. The effort force required to press the lever measured by digital force gauge is shown in Table 4. Force was measured from the first time the subjects started pressing on the lever until they pressed all the way before the force on the lever changed quickly. Device B required the most effort when pressing on the lever, which was reflected in the NRS score for the ease of pressing on the lever [Table 3-4]. Devices A, D, and E required equivalent force $(\mathrm{N})$. Devices $\mathrm{A}$ and $\mathrm{E}$ likely have the same pump structure as they are the same medication in a different volume. This result and exterior appearance of the devices strongly suggest that the pump structure for devices A and $\mathrm{E}$ is the same. In comparison, device $\mathrm{D}$ is a different medication sold from a different manufacturer. Although the exterior appearance is also different 
from that of devices A and E, the inner structure of the pump in reflected in the NRS score for ease of pressing on the lever for device $\mathrm{D}$ is likely the same as that in devices $\mathrm{A}$ and $\mathrm{E}$. This is also device $\mathrm{D}$, which was equivalent to that of devices $\mathrm{A}$ and $\mathrm{E}$.

Table 2: Correlations among age, pinch strength, grip strength, and hand/ finger size (Pearson's correlation coefficient) $(n=90)$

\begin{tabular}{|c|c|c|c|}
\hline \multicolumn{2}{|c|}{ vs. } & \multirow{2}{*}{$\begin{array}{c}\mathbf{r} \\
0.53\end{array}$} & \multirow{2}{*}{$\begin{array}{c}\mathbf{P} \\
<.0001\end{array}$} \\
\hline Pinch strength (kg) & Age(month) & & \\
\hline \multirow{2}{*}{ Grip strength (kg) } & Age(month) & 0.8 & $<.0001$ \\
\hline & Pinch strength(kg) & 0.576 & $<.0001$ \\
\hline \multirow{3}{*}{ Hand length from the wrist crease (mm) } & Age(month) & 0.797 & $<.0001$ \\
\hline & Pinch strength(kg) & 0.46 & $<.0001$ \\
\hline & Grip strength(kg) & 0.782 & $<.0001$ \\
\hline \multirow{4}{*}{ Thumb length (mm) } & Age(month) & 0.608 & $<.0001$ \\
\hline & Pinch strength(kg) & 0.365 & 0.0004 \\
\hline & Grip strength(kg) & 0.655 & $<.0001$ \\
\hline & Hand length from the wrist crease $(\mathrm{mm})$ & 0.724 & $<.0001$ \\
\hline \multirow{5}{*}{$\begin{array}{l}\text { Palm length from the wrist crease - thumb } \\
\qquad(\mathrm{mm})\end{array}$} & Age(month) & 0.526 & $<.0001$ \\
\hline & Pinch strength(kg) & 0.195 & 0.065 \\
\hline & Grip strength(kg) & 0.531 & $<.0001$ \\
\hline & Hand length from the wrist crease (mm) & 0.656 & $<.0001$ \\
\hline & Thumb length (mm) & 0.487 & $<.0001$ \\
\hline \multirow{6}{*}{$\begin{array}{l}\text { Palm length from the wrist crease - middle } \\
\qquad(\mathrm{mm})\end{array}$} & Age(month) & 0.662 & $<.0001$ \\
\hline & Pinch strength(kg) & 0.378 & 0.0002 \\
\hline & Grip strength(kg) & 0.655 & $<.0001$ \\
\hline & Hand length from the wrist crease (mm) & 0.87 & $<.0001$ \\
\hline & Thumb length (mm) & 0.585 & $<.0001$ \\
\hline & Palm length from the wrist crease - thumb (mm) & 0.658 & $<.0001$ \\
\hline \multirow{7}{*}{ Hand breadth (mm) } & Age(month) & 0.674 & $<.0001$ \\
\hline & Pinch strength(kg) & 0.474 & $<.0001$ \\
\hline & Grip strength(kg) & 0.695 & $<.0001$ \\
\hline & Hand length from the wrist crease (mm) & 0.767 & $<.0001$ \\
\hline & Thumb length (mm) & 0.626 & $<.0001$ \\
\hline & Palm length from the wrist crease - thumb (mm) & 0.48 & $<.0001$ \\
\hline & Palm length from the wrist crease - middle (mm) & 0.601 & $<.0001$ \\
\hline \multirow{8}{*}{ Hand circumference (mm) } & Age(month) & 0.695 & $<.0001$ \\
\hline & Pinch strength $(\mathrm{kg})$ & 0.502 & $<.0001$ \\
\hline & Grip strength(kg) & 0.784 & $<.0001$ \\
\hline & Hand length from the wrist crease (mm) & 0.735 & $<.0001$ \\
\hline & Thumb length (mm) & 0.623 & $<.0001$ \\
\hline & Palm length from the wrist crease - thumb (mm) & 0.476 & $<.0001$ \\
\hline & Palm length from the wrist crease - middle (mm) & 0.58 & $<.0001$ \\
\hline & Hand breadth (mm) & 0.759 & $<.0001$ \\
\hline
\end{tabular}

Table 3: Objective assessment by observers.

\begin{tabular}{|c|c|c|}
\hline Objective measures & Device & Comment (n) \\
\hline \multirow{4}{*}{ Subject can open the cap } & $\mathrm{C}$ & Not at all (1) \\
\cline { 2 - 3 } & $\mathrm{C}$ & Only on the second attempt (14) \\
\cline { 2 - 3 } & $\mathrm{C}$ & Only on the third attempt (3) \\
\cline { 2 - 3 } & $\mathrm{C}$ & On the third attempt out of 5 (2) \\
\cline { 2 - 3 } & $\mathrm{C}$ & Having difficulty (12) \\
\cline { 2 - 3 } & $\mathrm{D}$ & Having difficulty (1) \\
\hline
\end{tabular}




\begin{tabular}{|l|c|c|}
\hline \multirow{4}{*}{$\begin{array}{c}\text { Subject can hold the device with } \\
\text { three fingers }\end{array}$} & A & Barely able to hold with 3 fingers (12) \\
\cline { 2 - 3 } & A & Space in the lever is too tight for the finger (1) \\
\cline { 2 - 3 } & B & Barely able to hold with 3 fingers (5) \\
\cline { 2 - 3 } & B & Having difficulty holding with 3 fingers, but looks easier than A as its lever is wing-shaped (2) \\
\hline \multirow{3}{*}{\begin{tabular}{l} 
Subject can press on the lever \\
\cline { 2 - 3 }
\end{tabular}} & B & Not at all (1) \\
\cline { 2 - 3 } & B & Not on the first attempt (1) \\
\cline { 2 - 3 } & B & Having difficulty (12) \\
\cline { 2 - 3 } & B & Able to go halfway and having difficulty getting to the end (1) \\
\hline
\end{tabular}

Table 4: Effort force required to press on the lever of nasal drug delivery devices $(n=5)$.

\begin{tabular}{|c|c|c|}
\hline Device & Mean (SD) (N) & Range (N) \\
\hline A & $10.64(2.52)$ & $0.98-12.75$ \\
\hline B & $22.92(8.59)$ & $0.98-32.26$ \\
\hline C & $11.64(5.62)$ & $0.98-22.56$ \\
\hline D & $10.64(2.52)$ & $0.98-12.75$ \\
\hline E & $10.64(2.52)$ & $0.98-12.75$ \\
\hline
\end{tabular}
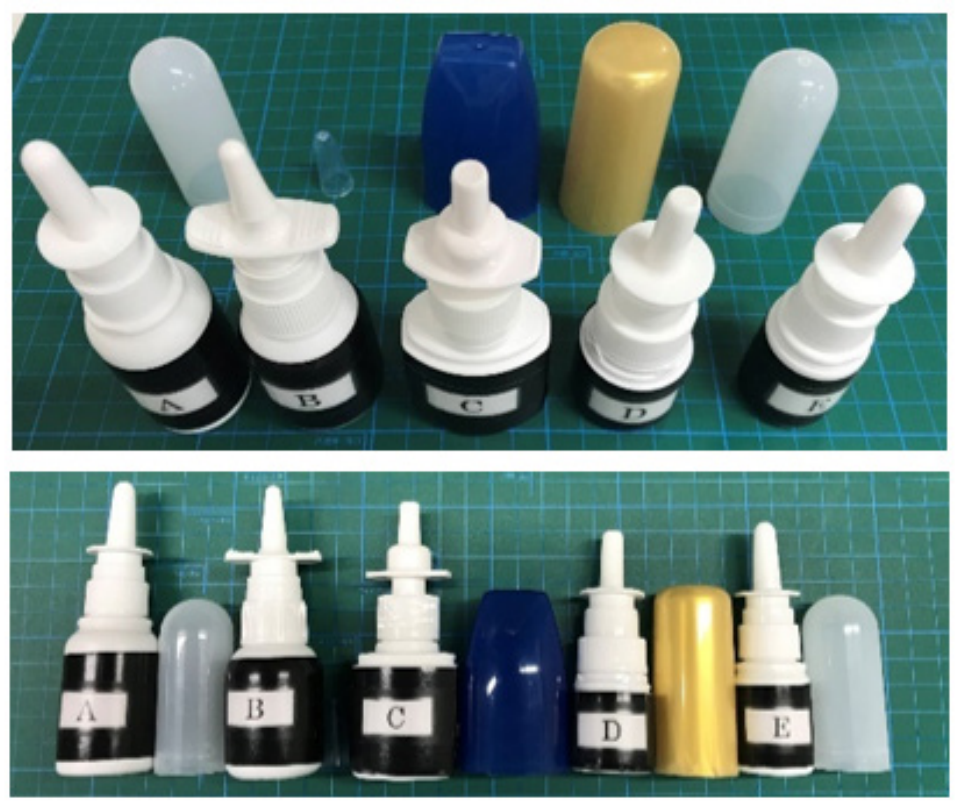

\begin{tabular}{cccc} 
Device & $\begin{array}{c}\text { Dose } \\
\text { (mL) }\end{array}$ & $\begin{array}{c}\text { Distance from bottom } \\
\text { to lever top }(\mathrm{cm})\end{array}$ & Lever shape \\
\hline A & 30 & 8.0 & Circle \\
B & 30 & 7.8 & Wing shape \\
C & 30 & 7.2 & Wing shape \\
D & 15 & 6.4 & Circle \\
E & 15 & 6.4 & Circle \\
\hline
\end{tabular}

Figure 1: Classification of the nasal drug delivery devices. 


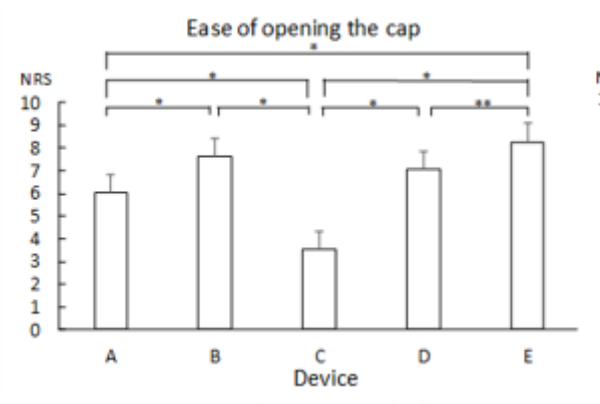

Ease of holding the device with three fingers
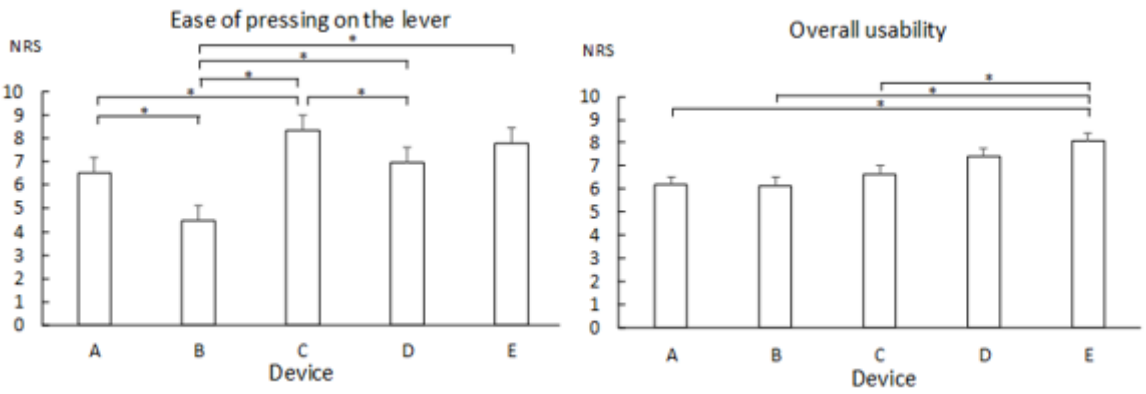

Figure 2: Usability of nasal drug delivery devices rated by study subjects $\left(n=90\right.$, mean $(S E)$, Steel-Dwass test, $\left.{ }^{* *} p<0.001,{ }^{*} p<0.01\right)$

\section{Usability of nasal drug delivery NRS devices with respect to the shape of the levers}

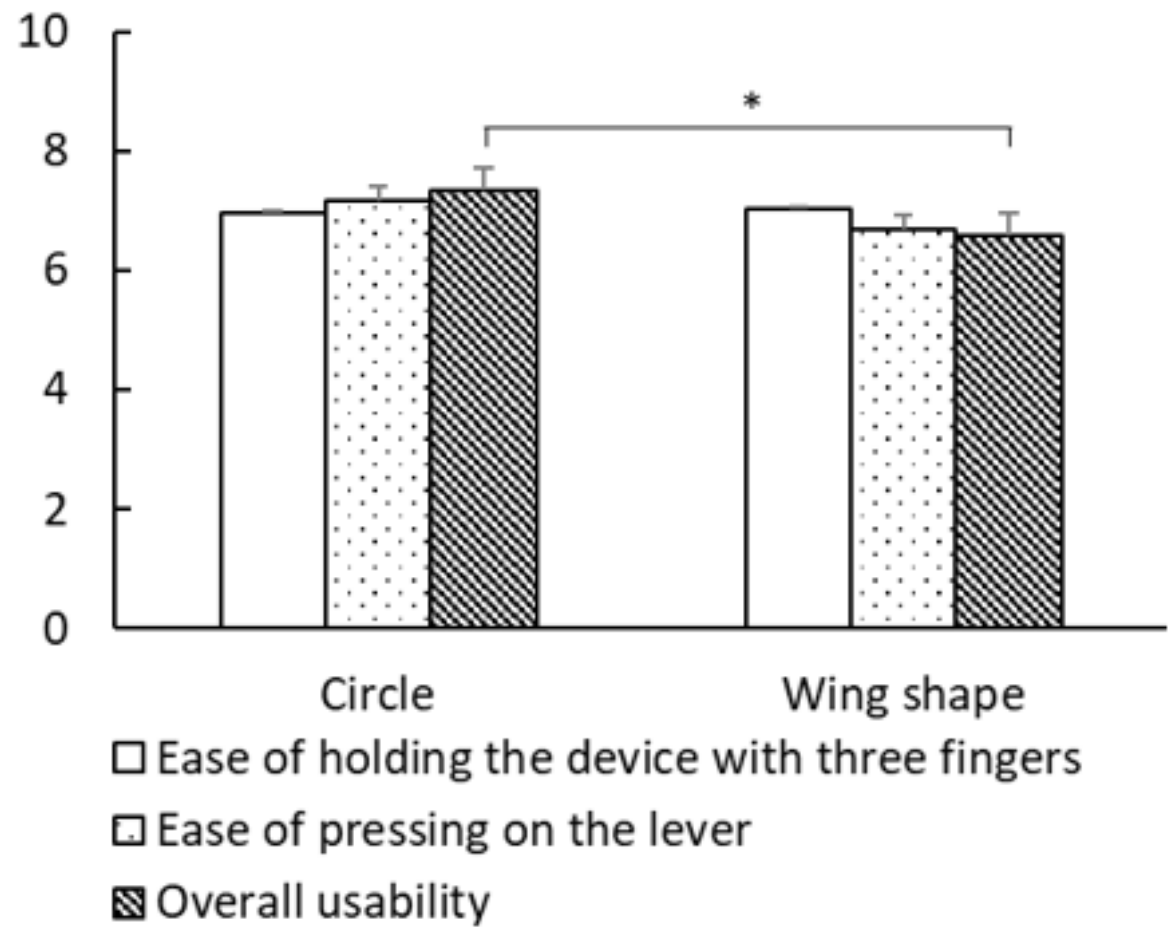

Figure 3: Usability of nasal drug delivery devices with respect to the shape of the levers (Circle $(n=270)$, Wing shape $(n=180)$, mean (SE), Wilcoxon rank sum test, $\left.{ }^{*} p<0.01\right)$ 


\section{Children}

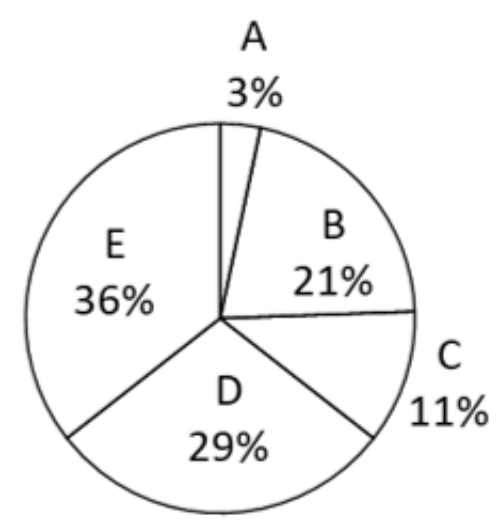

Parents

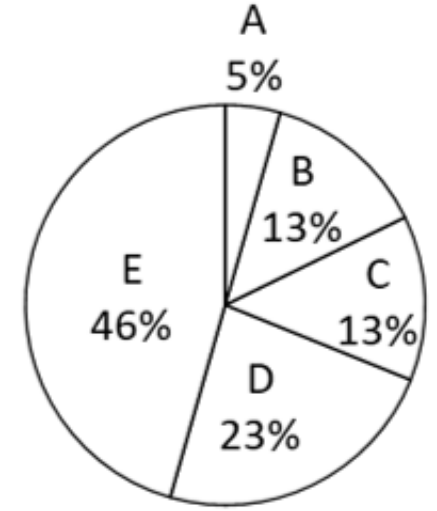

Figure 4: Nasal drug delivery devices selected for future usage $(n=90)$.

The correlations among age, pinch strength, grip strength, hand/ finger size, and each assessment metric for devices A-E (ease of opening the cap, ease of holding the device with three fingers, ease of pressing on the lever, and overall usability) are shown in Table 5. There was no correlation in any of the combinations. The difference in usability of the device based on the shape of the lever is shown in Figure 3. The NRS score for overall usability of was significantly higher for circular levers than for wing-shaped levers [Figure 2]. The correlation of each assessment metric with the distance from bottom to lever top and the average effort force required to press on the lever is shown in Table 6. For devices with circular levers, the ease of holding the device with three fingers was negatively correlated with the distance from bottom to lever top. Similarly, for devices with a wing-shaped lever, the ease of pressing on the lever was negatively correlated with the distance from bottom to lever top and the effort force for pressing on the lever. Subjects scored device $\mathrm{E}$ highest for their willingness to continue usage, followed by device D [Figure 4]. This result was consistent with the score for overall usability. Similarly, parents of the study subjects scored device $\mathrm{E}$ the highest for their children to continue usage, followed by device D [Figure 4]. Devices A and E, which are of the same medication in a different volume, were scored differently. Although they both had the same cap and likely the same pump structure, the distance from bottom to lever top among all the devices tested was the longest for device A and shortest for device E.

Table 5: Correlation of each assessment metric with pinch strength, grip strength, and hand/ finger size (Spearman's correlation coefficient).

\begin{tabular}{|c|c|c|c|c|c|c|c|c|c|c|c|}
\hline \multirow{2}{*}{\multicolumn{2}{|c|}{ vs. metric }} & \multicolumn{2}{|c|}{ A } & \multicolumn{2}{|c|}{ B } & \multicolumn{2}{|c|}{ C } & \multicolumn{2}{|c|}{ D } & \multicolumn{2}{|c|}{$\mathbf{E}$} \\
\hline & & $\mathbf{P}$ & rs & $\mathbf{P}$ & rs & $\mathbf{P}$ & rs & $\mathbf{P}$ & rs & $\mathbf{P}$ & rs \\
\hline \multirow{4}{*}{ Age (months) } & 1 & 0.048 & 0.655 & -0.155 & 0.146 & 0.116 & 0.276 & -0.214 & 0.043 & -0.137 & 0.197 \\
\hline & 2 & 0.206 & 0.051 & 0.121 & 0.256 & 0.126 & 0.237 & -0.064 & 0.551 & -0.022 & 0.835 \\
\hline & 3 & 0.145 & 0.172 & 0.078 & 0.463 & -0.132 & 0.217 & 0.052 & 0.626 & 0.015 & 0.891 \\
\hline & 4 & 0.197 & 0.063 & 0.066 & 0.54 & 0.089 & 0.404 & -0.034 & 0.752 & -0.103 & 0.339 \\
\hline \multirow{4}{*}{ Pinch strength (Kg) } & 1 & 0.075 & 0.485 & -0.038 & 0.725 & 0.125 & 0.24 & -0.299 & 0.004 & 0.034 & 0.752 \\
\hline & 2 & 0.183 & 0.084 & 0.001 & 0.99 & 0.109 & 0.306 & -0.173 & 0.104 & -0.079 & 0.461 \\
\hline & 3 & 0.113 & 0.289 & 0.143 & 0.178 & -0.193 & 0.068 & -0.151 & 0.156 & 0.01 & 0.929 \\
\hline & 4 & 0.163 & 0.124 & 0.177 & 0.097 & 0.106 & 0.319 & -0.164 & 0.122 & -0.042 & 0.699 \\
\hline \multirow{4}{*}{ Grip strength (Kg) } & 1 & 0.08 & 0.456 & -0.077 & 0.472 & 0.087 & 0.414 & -0.222 & 0.036 & -0.139 & 0.193 \\
\hline & 2 & 0.174 & 0.1 & 0.205 & 0.053 & 0.225 & 0.033 & -0.106 & 0.32 & -0.086 & 0.419 \\
\hline & 3 & 0.21 & 0.047 & 0.192 & 0.069 & -0.083 & 0.437 & -0.018 & 0.866 & -0.058 & 0.586 \\
\hline & 4 & 0.213 & 0.044 & 0.217 & 0.041 & 0.087 & 0.418 & -0.099 & 0.355 & -0.094 & 0.382 \\
\hline \multirow{4}{*}{$\begin{array}{l}\text { Hand length from the } \\
\text { wrist crease (mm) }\end{array}$} & 1 & 0.071 & 0.507 & -0.023 & 0.828 & 0.061 & 0.57 & -0.274 & 0.009 & -0.106 & 0.321 \\
\hline & 2 & 0.212 & 0.044 & 0.185 & 0.081 & 0.16 & 0.133 & -0.067 & 0.532 & -0.001 & 0.992 \\
\hline & 3 & 0.205 & 0.053 & 0.11 & 0.304 & -0.045 & 0.676 & 0.002 & 0.983 & 0.017 & 0.876 \\
\hline & 4 & 0.206 & 0.052 & 0.138 & 0.198 & 0.112 & 0.295 & -0.101 & 0.341 & -0.066 & 0.542 \\
\hline
\end{tabular}




\begin{tabular}{|c|c|c|c|c|c|c|c|c|c|c|c|}
\hline \multirow{4}{*}{ Thumb length (mm) } & 1 & -0.005 & 0.966 & -0.051 & 0.63 & 0.072 & 0.502 & -0.196 & 0.065 & -0.098 & 0.359 \\
\hline & 2 & 0.087 & 0.417 & 0.196 & 0.064 & 0.117 & 0.271 & 0.033 & 0.755 & -0.039 & 0.719 \\
\hline & 3 & 0.216 & 0.041 & -0.018 & 0.869 & -0.044 & 0.68 & 0.014 & 0.894 & -0.132 & 0.216 \\
\hline & 4 & 0.173 & 0.104 & 0.115 & 0.284 & 0.106 & 0.321 & -0.084 & 0.43 & -0.133 & 0.213 \\
\hline \multirow{4}{*}{$\begin{array}{l}\text { Palm length from the } \\
\text { wrist crease - thumb } \\
\text { (mm) }\end{array}$} & 1 & 0.008 & 0.943 & -0.13 & 0.224 & 0.152 & 0.153 & -0.137 & 0.197 & -0.23 & 0.029 \\
\hline & 2 & 0.142 & 0.181 & 0.209 & 0.048 & 0.181 & 0.088 & -0.108 & 0.31 & -0.115 & 0.281 \\
\hline & 3 & 0.05 & 0.64 & 0.113 & 0.288 & 0.005 & 0.96 & 0.021 & 0.843 & -0.049 & 0.648 \\
\hline & 4 & 0.085 & 0.424 & 0.112 & 0.296 & 0.183 & 0.084 & -0.073 & 0.493 & -0.021 & 0.849 \\
\hline \multirow{4}{*}{$\begin{array}{l}\text { Palm length from the } \\
\text { wrist crease - middle } \\
\text { (mm) }\end{array}$} & 1 & 0.062 & 0.564 & -0.05 & 0.641 & 0.081 & 0.447 & -0.182 & 0.086 & -0.106 & 0.321 \\
\hline & 2 & 0.216 & 0.041 & 0.151 & 0.157 & 0.138 & 0.195 & -0.01 & 0.925 & 0.019 & 0.863 \\
\hline & 3 & 0.183 & 0.085 & 0.094 & 0.376 & -0.065 & 0.541 & 0.055 & 0.609 & 0.021 & 0.848 \\
\hline & 4 & 0.166 & 0.118 & 0.074 & 0.49 & 0.064 & 0.552 & 0.016 & 0.878 & -0.029 & 0.784 \\
\hline \multirow{4}{*}{ Hand breadth (mm) } & 1 & -0.016 & 0.881 & 0.027 & 0.803 & 0.033 & 0.76 & -0.194 & 0.068 & -0.132 & 0.214 \\
\hline & 2 & 0.125 & 0.242 & 0.205 & 0.053 & 0.068 & 0.526 & -0.126 & 0.236 & 0.032 & 0.766 \\
\hline & 3 & 0.085 & 0.429 & 0.107 & 0.316 & -0.131 & 0.22 & -0.075 & 0.485 & 0.041 & 0.704 \\
\hline & 4 & 0.094 & 0.38 & 0.176 & 0.099 & 0.004 & 0.97 & -0.143 & 0.178 & -0.065 & 0.544 \\
\hline \multirow{4}{*}{$\begin{array}{l}\text { Hand circumference } \\
(\mathrm{mm})\end{array}$} & 1 & 0.071 & 0.504 & -0.033 & 0.754 & 0.061 & 0.568 & -0.156 & 0.141 & 0.002 & 0.985 \\
\hline & 2 & 0.223 & 0.035 & 0.287 & 0.006 & 0.183 & 0.085 & -0.019 & 0.86 & -0.001 & 0.996 \\
\hline & 3 & 0.18 & 0.09 & 0.125 & 0.241 & -0.066 & 0.537 & -0.004 & 0.971 & -0.036 & 0.739 \\
\hline & 4 & 0.223 & 0.034 & 0.179 & 0.094 & 0.057 & 0.592 & -0.041 & 0.704 & -0.074 & 0.493 \\
\hline
\end{tabular}

Table 6: Correlation of each assessment metric with the distance from bottom to lever top and average effort force required to press on the lever (Spearman's correlation coefficient)

\begin{tabular}{|c|c|c|c|c|c|c|c|}
\hline \multirow{2}{*}{\multicolumn{2}{|c|}{ vs. metric }} & \multicolumn{2}{|c|}{ All } & \multicolumn{2}{|c|}{ Circle $(n=270)$} & \multicolumn{2}{|c|}{ Wing shape $(n=180)$} \\
\hline & & \multirow{2}{*}{$\begin{array}{c}\mathbf{P} \\
-0.261\end{array}$} & \multirow{2}{*}{$\begin{array}{c}\text { rs } \\
<.0001\end{array}$} & \multirow{2}{*}{$\begin{array}{c}\mathbf{P} \\
-0.386\end{array}$} & \multirow{2}{*}{$\begin{array}{c}\text { rs } \\
<.0001\end{array}$} & \multirow{2}{*}{$\begin{array}{c}\mathbf{P} \\
0.128 \\
\end{array}$} & \multirow{2}{*}{$\begin{array}{c}\text { rs } \\
0.0863\end{array}$} \\
\hline \multirow{3}{*}{ Distance from bottom to lever top $(\mathrm{cm})$} & 2 & & & & & & \\
\hline & 3 & -0.184 & $<.0001$ & -0.11 & 0.0708 & -0.599 & $<.0001$ \\
\hline & 4 & -0.269 & $<.0001$ & -0.289 & $<.0001$ & -0.073 & 0.3302 \\
\hline \multirow{3}{*}{ Effort force on lever $(\mathrm{N})$} & 2 & 0.027 & 0.5631 & . & . & 0.128 & 0.0863 \\
\hline & 3 & -0.15 & 0.0015 & . & . & -0.599 & $<.0001$ \\
\hline & 4 & -0.18 & 0.0001 & . & . & -0.073 & 0.3302 \\
\hline
\end{tabular}

Table 7: Devices selected for future use and corresponding reasons.

\begin{tabular}{|c|c|c|}
\hline Device & Subjects & Parents \\
\hline \multirow{3}{*}{ A } & Easy to open the cap & Not too small \\
\hline & High volume & Contains a high volume of medication \\
\hline & Looks easy to put into the nose & Cap can be slightly tighter for carrying it around \\
\hline \multirow{3}{*}{ B } & Easy to hold & The shape of the lever is optimal for use \\
\hline & Easy to open the cap & Looks stable while pressing on the lever \\
\hline & Would be better if the cap was attached in order to not lose it & Looks easy to use, but worried about losing the cap \\
\hline \multirow{3}{*}{$\mathrm{C}$} & Cap is difficult to open, but it is easy to hold and press on the lever & Looks easy for children to use \\
\hline & Easy to press on the lever & The lever is sufficiently large and the cap is difficult to lose \\
\hline & Good shape & Looks like it does not roll around easily \\
\hline \multirow{5}{*}{$\mathrm{D}$} & Easy to hold & Looks easy to press on the lever \\
\hline & Easy to press on the lever & Good size \\
\hline & Easy to use & Looks easy for children to use \\
\hline & Small and optimal size & Each step of use seems smooth \\
\hline & Good size & Looks easy to open the cap \\
\hline
\end{tabular}




\begin{tabular}{|c|c|c|}
\hline \multirow{4}{*}{} & Easy to hold & D and E were both good, but prefer E for its transparent cap \\
\cline { 2 - 3 } & Easy to press on the lever & Small and looks easy to use \\
\cline { 2 - 3 } & Easy to open the cap & Good size for the hand \\
\cline { 2 - 3 } & Small and easy to use & Looks easiest to hold \\
\cline { 2 - 3 } & Easy to use & $\begin{array}{c}\text { Unlikely to lose the cap } \\
\text { Small and did not seem sanitary, A and E: E was better for chil- } \\
\text { dren's hands }\end{array}$ \\
\cline { 2 - 3 } & & Each step of use seems smooth \\
\cline { 2 - 3 } & & Looks easy to open the cap \\
\cline { 2 - 3 } & & Good overall \\
\cline { 2 - 3 }
\end{tabular}

Table 8: Medications used at home ( $n=37$, multiple responses provided as comments).

\begin{tabular}{|c|l|}
\hline Oral (allergy) & $\mathbf{5}$ \\
\hline Tablet (allergy) & 6 \\
\hline Powder (allergy) & 4 \\
\hline Nasal drops (allergy) & 5 \\
\hline Ointment, cream (allergy) & 1 \\
\hline Ointment (steroid) & 1 \\
\hline Ointment & 2 \\
\hline Oral (asthma) & 1 \\
\hline Powder (asthma) & 1 \\
\hline Patch (asthma) & 1 \\
\hline Inhalant (asthma) & 4 \\
\hline Oral & 7 \\
\hline Eye drops & 1 \\
\hline Tranquilizer (tablet) & 1 \\
\hline Trochiscus & 3 \\
\hline Cold remedy, antipyretic analgesic & 1 \\
\hline Intestinal medications & 2 \\
\hline Tablet & \\
\hline
\end{tabular}

Comments that were provided for devices that the subjects and their parents were willing to continue using are listed in Table 7. Devices D and E were described as easy to hold and press on the lever and having an optimal size. These characteristics demonstrate that devices D and E had a good balance of optimal structure and functionality for children to use. In contrast, devices $\mathrm{B}$ and $\mathrm{C}$ with a wing-shaped lever were described as having levers that are too large and difficult to press on. Furthermore, device B required the most effort to press on the lever. This suggests that the wing-shaped lever played a role in the unwillingness of the subjects and their parents to continue using the device. Based on the questionnaire administered to parents of the study subjects, 37 subjects use at least one Figure 3 medication at home (Table 8). Twenty-three of the subjects either previously used or are using medications in school on their own. None of the subjects were provided support from their teachers when taking medications
[Table 6]. Consistent with our previous small-scale study [11], one subject was unable to open the cap on device $C$ and another was unable to press on the lever on device B. This demonstrated that among OTC medications commonly available, there are some nasal drug delivery devices that children are unable to use even though they are of the appropriate age to use the device. In Japan, most children who are required to take medications in school do so by themselves. Indeed, our study demonstrated that the subset of study subjects who take medications at school were not provided with support from their schools.

Thus, it is essential that medications that are indicated for children be easy to self-administer. Physiological factors of subjects, such as age, pinch strength, grip strength, and hand/ finger size had little impact on the ease of opening the cap, ease of holding the device with three fingers, ease of pressing on the lever, or overall 
usability Figure 4. On the other hand, the shape of the lever affected overall usability. Furthermore, the distance from bottom to lever top for devices with circular levers was correlated with the ease of holding the device with three fingers. Similarly, for devices with wing-shaped levers, the distance from bottom to lever top and the average effort force required to press on the lever affected the ease of pressing on the lever. In addition to the average and maximum effort force, changes in the amount of force required during the action of pressing on the lever may impact the usability of a device [13]. Thus, it is important to further examine factors that affect usability while keeping other variables constant. A study performed in adults demonstrated that when pressing on the lever while holding the device with three fingers, the maximum force is applied by the thumb and in the index finger for circular and wing-shaped levers, respectively [13]. This suggests that the ease of pressing on the lever is affected by the shape of the device, specifically the shape of the base of the device for those with circular levers and the shape of the lever for those with wing-shaped levers, where it encounters the fingers that apply the maximum force. Future studies should examine the impact of contact force, although the measurement of contact force can be challenging in children Table 7.

\section{Conclusion}

In the present study, we examined the usability of nasal drug delivery devices and factors that affect the usability. In Japan, it is common for consumers to purchase OTC medications by simply reading the product labeling. Furthermore, pharmacists that work at typical drug stores do not have opportunities to learn about the specific characteristics of each drug delivery device that is available for OTC medications. Based on our study, marketing for OTC medications should consider the significant effects of usability on the choice of medication and that devices may not be easy to use for children. Furthermore, it is important to design a user-friendly device for OTC medications Table 8

\section{Conflict of Interest}

There are no conflicts of interest influencing the work reported in this paper.

\section{Acknowledgment}

This work was supported by JSPS KAKENHI Grant Number JP18K02464.

\section{References}

1. (2018) Estimates of National Medical Care Expenditure, Health Statistics Office, Ministry of Health and Welfare (MHLW)

2. (2018) Statistics of Production by Pharmaceutical Industry, Economic Affairs Division, Ministry of Health and Welfare (MHLW).

3. (2019) Health at a Glance: OECD Indicators, Organisation for Economic Co-operation and Development (OECD).

4. (2020) Annual Health, Labour and Welfare Report 2020, Ministry of Health and Welfare (MHLW).

5. The self-medication tax system (special exceptions for medical expense deductions), Ministry of Health and Welfare (MHLW).

6. Sadamoto K, Murata M, Kubota K (2016) Life and Medication-Needs for Packaging and Devices. Int J Pharm Pharm Res 8(1): 232-243.

7. Guidance for Industry (1999) “Container Closure Systems for Packaging Human Drugs and Biologics". FDA.

8. Osterberg L, Blaschke T (2005) Adherence to Medication. N Engl J Med 353: 487-497.

9. Hanya M, Shibata A, Kamei H, Matsuba K, Asai M, et al. (2007) Analysis of Pharmacist Communication with Patients in Triaging OTC Drugs. J Pharm Health Care Sci 33(8): 693-701.

10.(2015) Pharmaceutical Administration and Regulations in Japan, Regulatory Information Task Force Japan Pharmaceutical Manufacturers Association, Japan.

11. Murata M, Sumi M, Kubota K, Sadamoto K (2017) Usability of intranasal drug delivery devices: Children and their Parents. Int. j. res. pharm pharm. sci 2(6): 1-5.

12. Kouchi M (2012) AIST- Data on the dimensions of the Japanese hand. Digital Human Research Team, Artificial Intelligence Research Center, National Institute of Advanced Industrial Science and Technology (AIST).

13. Murata M, Sumi M, Kubota K, Hayashi M, Sadamoto K. Usability evaluation of intranasal drug delivery devices and Ergonomics. Jpn J Ergonomics 55 Supp.: S1G1-2. 